CORRECTION

\title{
Correction: Force field generalization and the internal representation of motor learning
}

\section{Alireza Rezazadeh, Max Berniker}

The images for Figs 1 and 2 are incorrectly switched. The image that appears as Fig 1 should be Fig 2, and the image that appears as Fig 2 should be Fig 1 . The figure captions appear in the correct order.

\section{fopenaccess}

Citation: Rezazadeh A, Berniker M (2020) Correction: Force field generalization and the internal representation of motor learning. PLOS ONE 15(1): e0227963. https://doi.org/10.1371/ journal.pone.0227963

Published: January 16, 2020

Copyright: ๑ 2020 Rezazadeh, Berniker. This is an open access article distributed under the terms of the Creative Commons Attribution License, which permits unrestricted use, distribution, and reproduction in any medium, provided the original author and source are credited. 

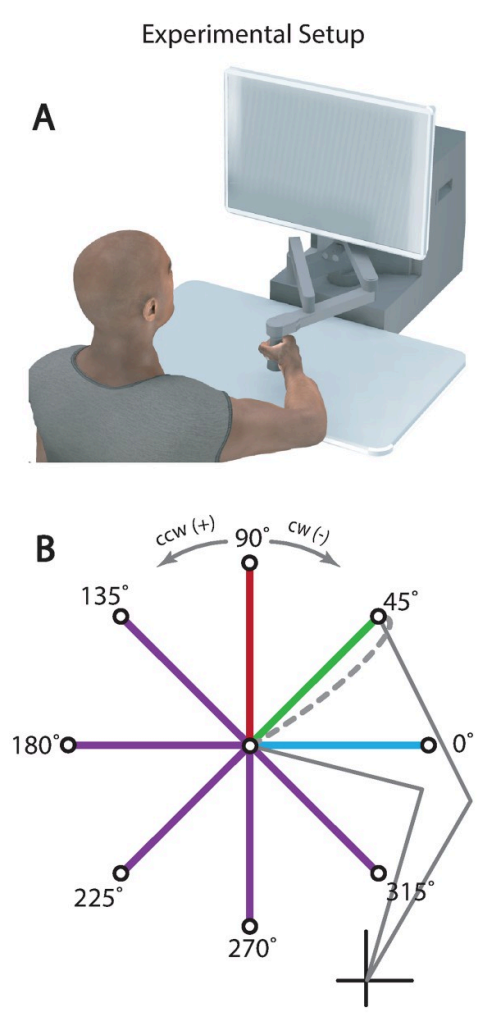

Representation of the FF Strength

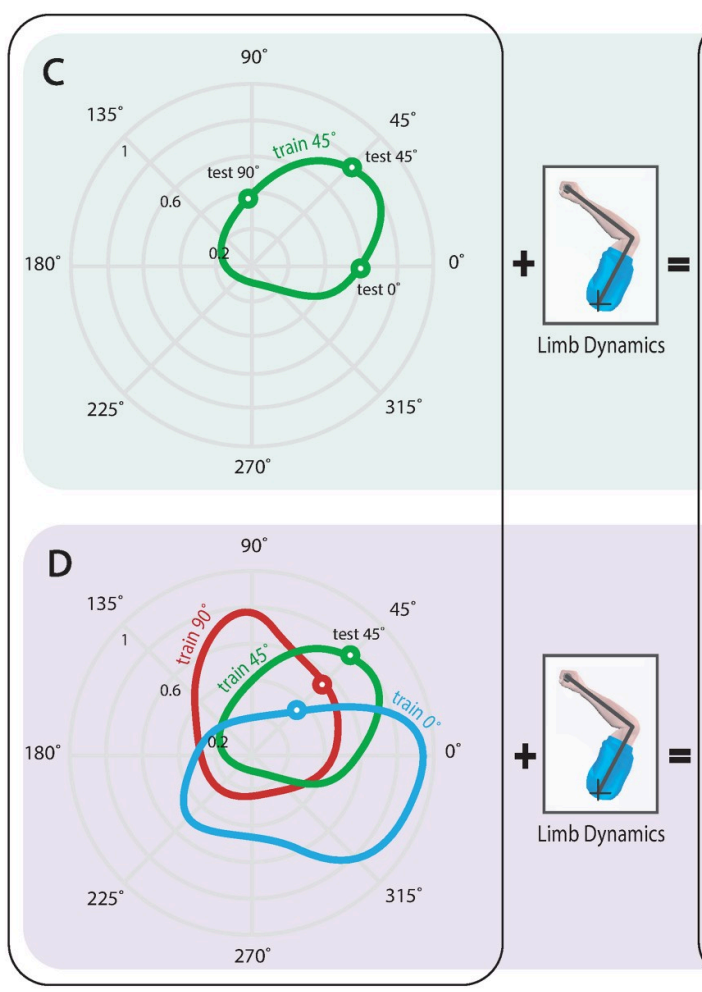

Generalization

(Measured in Error-Clamp Trials)

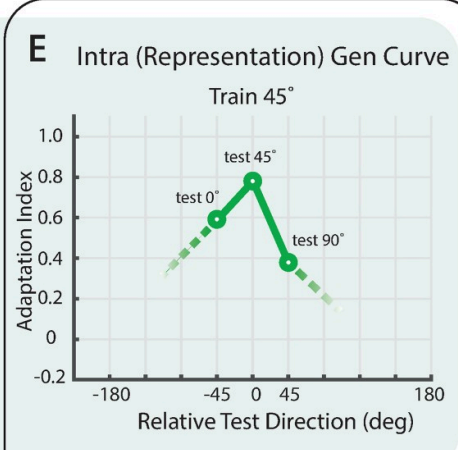

F Inter (Representation) Gen Curve Test $45^{\circ}$

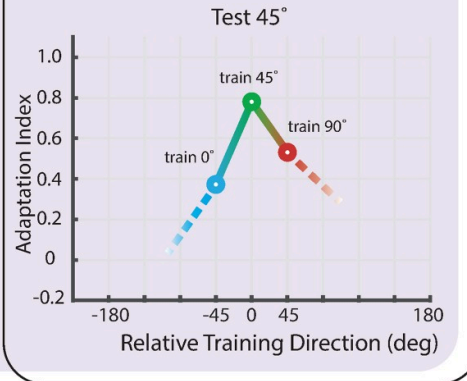

Fig 1. Experimental setup and generalization curves. (A) Subjects made reaches in a null field, a curl field or an error-clamp. After landing on target the robot brought their hand back to the center position. (B) Eight standard targets were located 10- $\mathrm{cm}$ away from a home target. Each of eight groups practiced reaching in a CW force field towards one of the eight targets (the gray dashed line depicts a reach in the field to the $45^{\circ}$ target) and then tested for generalization to all remaining targets using error-clamp trials. (C) A schematic of the internal representation of the learned force field for the $45^{\circ}$ target group. The curve depicts a subject's estimated strength of the force field in polar coordinates (green contour). (D) Using this estimate the limb makes a generalization reach. Combining the measurements made from many such reaches after training on the $45^{\circ}$ target constitutes an intrarepresentation generalization curve (green circles). These measurements are influenced by both the force field estimate and changes in limb impedance with reach direction. (E) Schematic of representations from three groups of subjects learning the field for the $0^{\circ}$ (blue contour), $45^{\circ}$ (green contour), and $90^{\circ}$ (red contour) targets. (F) Combining the measurements made across all three groups when reaching to the same $45^{\circ}$ target constitutes an intrarepresentation generalization curve. This curve corrects for the changes in reach direction.

https://doi.org/10.1371/journal.pone.0227963.g001 
A

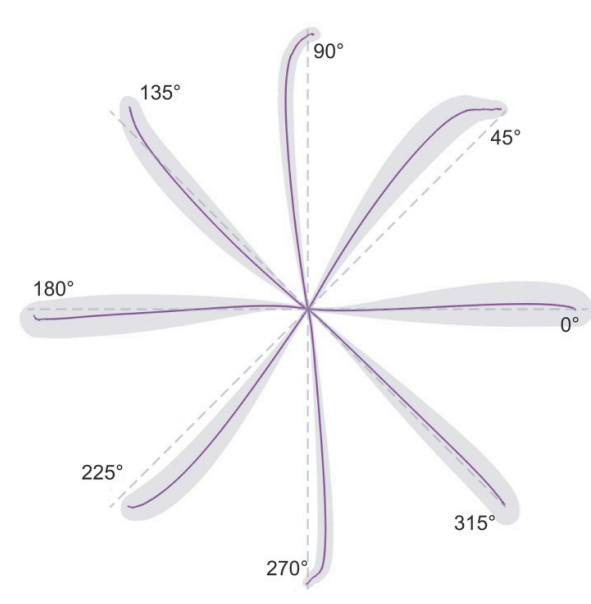

B

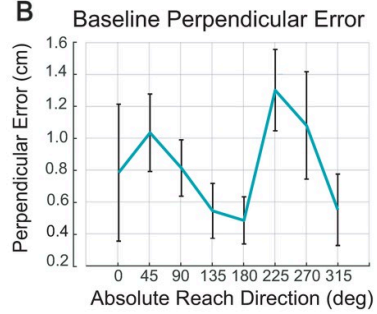

D Baseline Adaptation Index

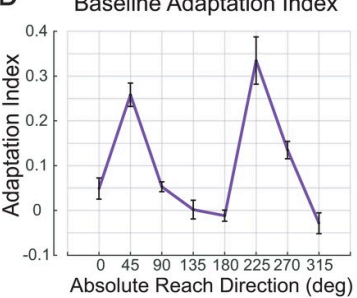

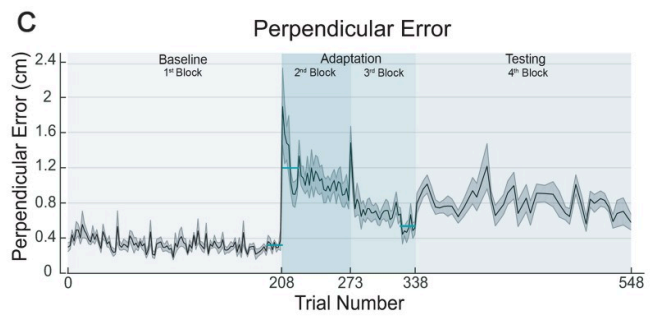

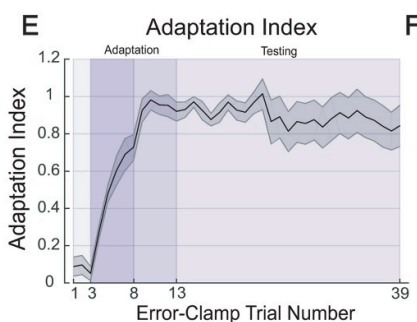

Early Adaptation PE

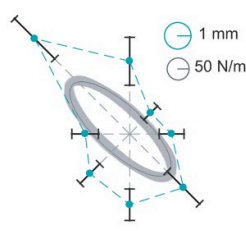

Fig 2. Experiment's behavioral results. (A) Baseline reaches in null field made with visual feedback (across-subject averages \pm SEM). Reaches slightly deviate from a straight path (gray dashed lines). (B) Baseline perpendicular errors averaged across subjects. (C) Across-subject average perpendicular errors $( \pm$ SEM) are plotted across all trials of the experiment. Only error-clamp trials are excluded. Subjects practiced reaching in force field during the $2^{\text {nd }}$ and $3^{\text {rd }}$ blocks with a brief rest between. (D) Baseline adaptation index averaged across subjects. Note the qualitatively similar shape to the perpendicular errors in B. (E) Force field learning curve averaged across all subjects and all reach directions. Mean values to the training targets $( \pm$ SEM) are plotted across error-clamp trials. (F) Early adaptation perpendicular errors (mean \pm SEM of first 20 trials) are averaged across subjects and overlaid on a hand stiffness ellipse from [7]. Note the qualitatively similar shapes of errors and hand stiffness.

https://doi.org/10.1371/journal.pone.0227963.g002

\section{Reference}

1. Rezazadeh $A$, Berniker $M(2019)$ Force field generalization and the internal representation of motor learning. PLoS ONE 14(11): e0225002. https://doi.org/10.1371/journal.pone.0225002 PMID: 31743347 